\title{
Weapon Design Patterns in Shooter Games
}

\author{
Robert Giusti, Kenneth Hullett, Jim Whitehead \\ Augmented Design Lab \\ University of California, Santa Cruz \\ Santa Cruz, CA \\ rgiusti@ucsc.edu, \{khullett, ejw\}@soe.ucsc.edu
}

\begin{abstract}
With the increasing complexity of interactive games, the need for a common vocabulary to describe patterns grows. Action-oriented games focus heavily on players using weapons, but categorizations used for weapons are borrowed from real-world patterns, classifying them according to the physical innerworkings of the weapons rather than their gameplay effects. This terminology draws lines between similar weapons that elicit similar gameplay and creates unnecessary distinctions between fictional and non-fictional weapons. We aim to classify weapons in a way that defines weapons by the gameplay behaviors they elicit using a language of common weapon design patterns. This paper expands the taxonomy of game design patterns to better describe how weapons can be used to influence gameplay. These patterns act as a language for communicating game design concepts.
\end{abstract}

\section{Categories and Subject Descriptors \\ K.8 [Personal Computing]: Games.}

\section{General Terms}

Design, Human Factors

\section{Keywords}

game design, design patterns.

\section{INTRODUCTION}

Digital game design suffers from a lack of a common language within the community of designers, making it difficult to describe or teach about game design. Through design patterns we create terminology for describing the recurring concepts that appear across a range of designs. Weapon design is currently obstructed by reliance on terminology used to describe real-life weapons.

To define and discuss weapons, game and level designers have repurposed an existing classification system: the terminology used to refer to real-life weapons, terms such as "Sub-machine Gun" and "Sniper Rifle." Though these classifications do easily explain the mechanics of the weapon, the use of such terminology fails to accurately describe gameplay behaviors and to encompass the fictional aspects of digital games.

Knowing how a particular weapon functions in real life does not actually give an accurate depiction of how the weapon functions within a game. For example, the shotgun in Halo has a much shorter effective range than its real-life counterpart. Many similar

Permission to make digital or hard copies of all or part of this work for personal or classroom use is granted without fee provided that copies are not made or distributed for profit or commercial advantage and that copies bear this notice and the full citation on the first page. To copy otherwise, or republish, to post on servers or to redistribute to lists, requires prior specific permission and/or a fee.

$D P G$ 2012, May 29, 2012, Raleigh, North Carolina, USA.

Copyright 2012 ACM 978-1-4503-1854-9/12/05 ... \$15.00. weapons fall into different weapon patterns depending on how designers implement them.

Though many action and adventure games use weapons, shooter games are affected by this lack of terminology more than others due to the fact that weapons are at the core of gameplay. In the vast majority of first-person shooters, the player's weapon never even leaves their view. In addition, weapons are the central method through which players interact with the world in these games.

With this pattern collection we hope to create a language that can be used to describe weapons in a way that encapsulates the gameplay behaviors that each pattern elicits. Each pattern is named in a way that aims to be inclusive of all weapons, fictional or nonfictional, that elicits similar player behavior.

Further, we hope that by creating a vocabulary with which designers can better discuss how to use these game objects in order to meet their design goals, we can make it easier to share ideas and foster the communication of professionals and the education of those entering the field.

We accumulated these patterns through analyzing weapons in popular and historically significant first- and third-person shooter games.

\section{RELATED WORKS}

Though much work has been done to identify design patterns for games, most of it has been at a high level and not oriented towards weapon design. Björk and Holopainen [1] were among the first to apply the idea of design patterns to video game design, and their formal system of design patterns laid the groundwork for the field. However their patterns cover a wide breadth of aspects of game design and therefore lack depth on particular subjects. Hullett and Whitehead [3] and Rivera et al. [5] have done work defining patterns in-depth for level design and non-player character design respectively, but there still remain many fields for which a deeper understanding of the underlying effects of design could be better categorized.

In weapon design for games, some research has been done into how weapons can be used to affect pacing of a game and a player's sense of progression. Larsen [4] suggests that weapons can be used to instill a sense of artificial character development. Weir [5] argues that weapon design can guide the pacing of the game while encouraging the player to develop their own skills.

\section{WEAPON PATTERNS}

To provide a basis for defining patterns in weapon design, the following template will be used:

Name - A descriptive identifier used to refer to the pattern that is recognizable and imparts the core functionality of the pattern.

Description - A brief explanation of the typical features of a weapon derived from this pattern. 
Affordances - Aspects of the pattern that can be varied between different weapons within the pattern.

Consequences - How use of the weapon pattern affects gameplay.

Level Patterns - Relationships between the weapon pattern and patterns in level design. These patterns are borrowed from Hullett, and include the following:

- Sniper Location - A protected, elevated location that overlooks some portion of the level.

- Gallery - An elevated area parallel and adjacent to a narrow passageway.

- Arena - An open area or wide corridor.

- Stronghold - A confined area with good cover and limited access points.

- $\quad$ Split Level - A corridor with an upper and lower section, where those on the upper section can attack those on the lower section.

- Hidden Area - A small area off the main route that contains items for the player.

- Flanking Route - An alternate path that allows characters to gain positional advantage.

- $\quad$ Turret - An area with a high-powered weapon where one side has a clear advantage.

- Vehicle Section - Sections of alternate gameplay where the player drives or rides in a vehicle.

NPCs - Relationships between the weapon pattern and patterns in non-player character design. These patterns are borrowed from Rivera's work, and include the following patterns and subpatterns:

- $\quad$ Soldier - An NPC that pressures the player from range.

- Grunt - A weak enemy that attacks from a medium distance, often in groups.

- Elite - A strong enemy that works to contain the player from a medium distance.

- Grenadier - A weaker enemy that maintains long distance to encourage players to move forwards.

- Sniper - An enemy that deals high damage from a long distance to force players to move carefully.

- Aggressive - An NPC that attempts to close the distance between itself and its target in order to increase pressure.

- Suicidal - An enemy that immediately rushes at the player, at the cost of its own life.

- Swarm - An enemy that rushes the player in groups, but deals low damage individually.

- Berserker - A strong NPC that deals a high amount of damage over a prolonged amount of time.

- Carrier - An NPC that will spawn more NPCs during an encounter.

- Sacrificial - An NPC that creates more NPCs in the case of its own death.

- Summoner - An enemy that spawns more NPCs at a distance
- Tank - An NPC that poses a significant singular threat and prevents the player from proceeding.

- Turret - A slow-moving NPC that deals high damage at a long range.

- Shielded - An NPC with a large amount of armor, but only in a single direction.

Examples - Uses of the weapon design pattern from popular commercial shooter games.

Patterns contained within another are considered to be super- or sub-patterns of each other. Patterns are not mutually exclusive from each other; a weapon can fit multiple weapon patterns.

The patterns defined could be considered sub-patterns of Björk's Weapon pattern [6]. A large number of affordances can be considered universal among weapon patterns, including:

- How much damage the weapon deals

- The range of the weapon

- The area of effect of the weapon

- How often the weapon can be used ("Cooldown")

- How many times the weapon can be used before needing to be reloaded ("Capacity")

- How much ammunition a player can carry

- How carrying the weapon affects the player's movement

- How the weapon imparts damage to the enemy (On hit, delayed, continuous, etc.)

- Any special effects that the weapon has on the enemy

- Any special abilities that the weapon bestows

Repetition of a Universal Affordance within a particular pattern description signifies that pattern differs significantly within the pattern in that aspect.

\subsection{Sniping Weapon}

Description - A weapon for engaging enemies from a long distance. These weapons do large amounts of damage per shot, have some sort of vision magnification system, and aim accurately. Sniping Weapons often reveal the player's location, hold limited amounts of ammunition, or require an extended reload period.

Affordances -

- The amount of view magnification that the weapon gives

- Additional difficulty in aiming the weapon accurately

- Any cues that reveal the player's location

Consequences - Sniping Weapons encourage the player to maintain distance from enemies, engaging from long-range. They push players towards cover, which protect from groups of enemies.

Level Patterns - Players with a Sniping Weapon fare best in Sniper Locations, which offer both good cover and a good view of locations where targets might be.

NPCs - Sniper enemies are defined by having Sniping Weapons, however Turrets and Elites also sometimes utilize Sniping Weapons. Such enemies pressure players from a distance, forcing them to search for alternate routes. 
Sniper Weapons allow a player to eliminate powerful Tanks and carriers without engaging them directly, but are less effective against Grunts and Rush enemies who attack in groups.

Examples - An example of a Sniping Weapon is the Sniper Rifle in Halo 3. The weapon offers view magnification, has a capacity of four shots that can be fired in succession, and leaves a visible trail along a fired bullet's path to reveal the player's location.

In Ratchet and Clank: Up Your Arsenal, the Sniping Weapon has the added drawback of pulling the player from third-person perspective into first-person perspective and prohibiting the player from moving while in this view. The weapon balances itself out by dealing a very high amount of damage per shot.

The Huntsman from Team Fortress 2 functions as a much different Sniping Weapon, allowing the player to trade in their guns and bullets for a bow and arrow. Since the weapon offers no view magnification to the user, it could be mistaken for an Assault Weapon, but the slow firing rate along with its difficulty in aiming and high damage per shot force the user into the same gameplay behaviors as the user of a sniper rifle.



Figure 1: A player using a Sniping Weapon in Halo 3

\subsection{Close Blast}

Description - A Close Blast weapon fires in a quick and inaccurate manner as a means of hastily eliminating one or few enemies at a close range with a large area of effect. The large area of effect reduces the need for high accuracy. Shotguns, flamethrowers, and some submachine-guns are Close Blast weapons.

Affordances -

- Whether the weapon sends out single shots that diminish in strength over distance or multiple simultaneous shots that can each hit the target individually

- Whether the weapon is capable of hitting multiple targets in a single blast.

Consequences - When given a Close Blast weapon, a player tends to use cover as much as possible. These weapons cause players to move tactically and strategically, minimizing distance in firefights before starting them.

Level Patterns - Close Blast weapons are best fitted to Choke Points, where a player can engage targets at short range. Arenas with lots of cover are also advantageous.
Least preferable to Close Blast users are Arenas or Galleries without enough cover to protect the player from mid- and longrange attacks.

NPCs - Close Blast weapons are often found in the hands of Berserker and Elite enemies, who seek to tactically catch the player in close-quarters.

A Close Blast weapon's area of effect is advantageous against Swarm and Carrier enemies, since the player can quickly eliminate targets in close vicinity to each other. Close Blast weapons are unable to harm protected enemies such as Snipers and Turrets.

Examples - The shotgun from Goldeneye 007 offers a prime example of a Close Blast weapon. At close range, the weapon has a large area of effect, but as distance increases the effectiveness of the weapon quickly drops off.

Though mechanically and aesthetically very different, a submachine-gun such as the one found in Half-Life 2 functions very similarly to a shotgun. The weapon fires through a single clip very quickly, firing off the shots in an inaccurate cone. At a close range, a skilled player may be able to confidently engage two, maybe three, soldiers before being forced to reload, while at medium range the weapon might not kill just one enemy with an entire clip of bullets.

In Team Fortress 2, the Pyro class has a flamethrower, which fires a continuous stream of fire over a short distance. The weapon damages the enemy most when the player stays within the close range, and the weapon continues to deal a small amount of damage over time as long as the enemy stays on fire.

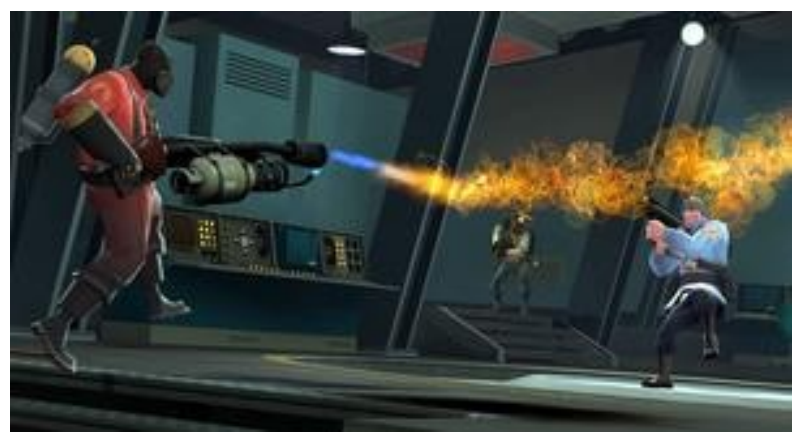

Figure 2: A Pyro using a flamethrower in Team Fortress 2

\subsection{Assault Weapon}

Description - Mid-range weapons that fire fairly accurately and quickly, but deal low amounts of damage per shot. Many games contain multiple different types of Assault Weapons to fit different scenarios or player preferences.

Affordances -

- Whether the player can fire the weapon continuously ("Automatic"), must fire shots individually ("SemiAutomatic"), or has a mode that fires several shots in quick succession ("Burst-fire")

- Whether the player can change affordances of the weapon within the game.

Consequences - Due to their high firing rate, Assault Weapons heighten the pace and excitement of a level. Given their versatility, skilled players can confidently move through areas with little to no cover. 
Level Patterns - Assault Weapons perform well in Arenas and Split Levels, since the user can easily switch between targets in different directions.

NPCs - Grunts and Elites often use Assault Weapons to pester the player from a distance, dealing small enough damage to be a threat but allowing the player time to react tactically.

Players can use Assault Weapons to easily combat Swarm enemies and Grunts, who often show up in large numbers. Assault Weapons allow the player to quickly take out low-threat enemies.

Examples - Call of Duty 4: Modern Warfare 2 gives players a variety of choice in Assault Weapons, showcasing the effects of slight modifications to an Assault Weapon's affordances. For example, the M4A1 is accurate and fires continuously, but deals low damage, while the FAMAS fires in bursts but reloads slowly.

The Halo series also contains a variety of Assault Weapons. The plasma rifle shoots a fast stream of energy bolts accurately at the player's aim. Instead of limiting shots per magazine, the weapon has a cool-down meter that must be managed, or else the weapon overheats and cannot be fired for a short period.

\subsubsection{Sidearm}

Description - A weak Assault Weapon, usually used as filler until the player obtains a better weapon. These deal low damage but have large quantities of ammunition. Sidearms slow down the pace of the level and heighten challenge and tension.

\section{Affordances -}

- Whether the weapon uses any ammunition system or is an infinitely reliable backup weapon.

- Whether the weapon takes up space in the player's inventory or is always available.

Consequences - Since the player is at a disadvantage to normal enemies, he or she will proceed cautiously and search the environment for better weapons. Forcing the player to fight powerful NPCs with only a Sidearm drastically increases tension.

Level Patterns - A player with only a Sidearm will utilize cover and Choke Points to their advantage. The player is, however, vulnerable to enemy Sniper Locations and weak in Arena firefights.

NPCs - Grunts with Sidearms often litter the battlefield in order to give the player easy targets, or to ease the player into the game early on.

When a player has a Sidearm, they are generally at a disadvantage against all enemies, and therefore they are more inclined to retreat from large threats and focus on taking on even Grunts tactically.

Examples - In Half-Life 2, the player is given a light pistol that does not do much damage, but ammo for it is plentiful throughout the game. If the player runs out of other weapons, they can revert to the pistol while they search for more ammo for other weapons.

Halo 3 includes several Sidearm weapons, such as the pistol and plasma rifle, that do little damage on their own. In this game, however, two Sidearms can be wielded simultaneously, allowing the player to use the weapons more effectively as Assault Weapons. Wielding two weapons at once also has the drawback of prohibiting the player from using their grenade Thrown Projectiles, which are always usable when only using a single weapon.

\subsection{Projectile}

Description - Objects thrown or fired in a physics-defined arch. Most often, Projectiles are explosives that deal damage in a large area of effect. Projectiles are also associated with long reload times and small capacities. Projectiles also often have a low amount of maximum ammunition.

\section{Affordances -}

- The range of the weapon

- If the effect is immediate or delayed

- The area of effect of the weapon

- Any special effects of the weapon

Consequences - Projectile weapons are useful for circumventing cover. Also, they heighten the challenge through being more difficult to aim than other weapons.

Level Patterns - Projectiles can be used to harm enemies in Sniper Locations or guarding Choke Points without directly engaging them. Players using Projectiles are often vulnerable to Split Levels and Galleries, due to ammunition limitations and a lack of sufficient cover.

NPCs - Grenadiers, Elites, and sometimes Tanks use Projectiles to force the player out of cover and impose a greater threat.

Projectiles allow players to take on large groups of enemies, such as Swarms and Carriers, and fight against heavy enemies, such as Tanks and Snipers, without engaging them directly. The long recharge times and tendency for Projectiles to have large areas of effect make them less effective against Berserkers and Suicidals.

Examples - The Demoman class from Team Fortress 2 has a Grenade Launcher that allows the player to fire pipe bombs at enemies. These pipe bombs explode on impact with an enemy; otherwise the bombs roll for a few seconds before exploding.

In the Halo series, the rocket launcher is a weapon that is both a Launched Projectile and Power Weapon. The weapon launches a rocket at high velocity, creating a large explosion that can instantly kill targets, both those on foot and those in vehicles. However, the weapon carries very limited ammunition and takes up space in the player's limited arsenal.

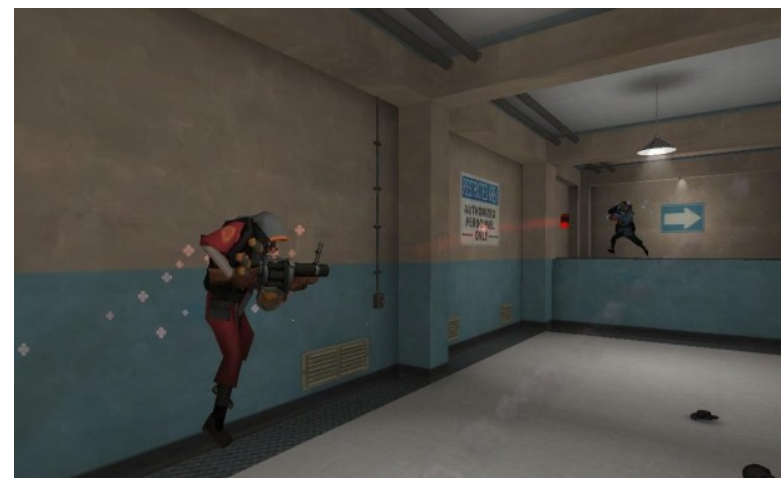

Figure 3: A player firing Projectiles in Team Fortress 2

\subsubsection{Thrown Projectile}

Description - A non-bullet object thrown by the hand of the player's character and categorized by short range and highly affected by gravity. Thrown Projectiles often have high damage or severe special effects, balanced by scarce ammunition. 
- Special effects associated with the physical object of the projectile

Consequences - The player is able to attack opponents who are behind cover, however they are forced to keep in mind their ammunition and range limitations.

Level Patterns - Thrown Projectiles allow players to defeat an enemy guarding a Choke Point, or players on another level of a Split Level. In areas with long distances, such as Sniper Positions, or with enemies at multiple angles, such as Arenas and Flanking Routes, Thrown Projectiles are not very effective.

NPCs - Elites utilize Thrown Projectiles in order to pressure players who are taking cover. Some Summoners use their spawned units as a sort of Thrown Projectile as a way of deploying them.

A player can use Thrown Projectiles much like normal Projectiles to attack heavy Tanks from behind cover. Thrown Projectiles are often more effective against solitary, close-range targets and less effective against loosely grouped Swarm and Grunt enemies.

Examples - In Call of Duty 4: Modern Warfare 2, the throwing knife is a powerful Thrown Projectile with harsh limitations. The weapon has a short range, however a hit with the knife immediately kills the enemy. A player also may only carry one knife at a time.

Halo 3 offers players a handful of varied thrown projectiles. Fragmentation grenades can be thrown a good distance and rebound off any obstacles until they detonate after a set amount of time. Players also have the option of using plasma grenades instead, which attach themselves to level geometry and players on contact, but have a shorter range and smaller blast radius.

\subsection{Power Weapon}

Description - A weapon that gives the player a clear advantage over other available weapons by either being incredibly powerful or by bestowing unique abilities. Major drawbacks may be present in order to balance the weapon against its obvious advantages.

Affordances -

- Special abilities that the player is bestowed with

- Drawback for using the weapon

- Circumstances for obtaining the weapon

Consequences - Power Weapons increase pace and decrease tension in order to allow for the designer to create moments of low challenge. Power Weapons give the player a sense of equivalency when facing a vehicle or boss enemy.

Level Patterns - Power Weapons might be a reward for players who explore to find Hidden Areas, or give players a fairer fight in otherwise overwhelming situations, such as Strongholds.

NPCs - Power Weapons are usually limited to Tanks, but can also make Elites and Berserkers especially threatening to the player.

A player with a Power Weapon can quickly eliminate most enemies without much challenge. Elites and Tanks may be given ways to exploit the drawbacks of the player's Power Weapon.

Examples - In Halo 3, the Spartan Laser fires a powerful laser beam that instantly destroys anything in its path. It requires several seconds of "charge-up" time, during which it gives off a loud sound that reveals the player.

Call of Duty 4: Modern Warfare 2 presents Power Weapons in a very different manner, through "Killstreak Rewards." These are given to players as rewards for achieving a certain number of kills in a single life, fueling the momentum of a successful player or team.

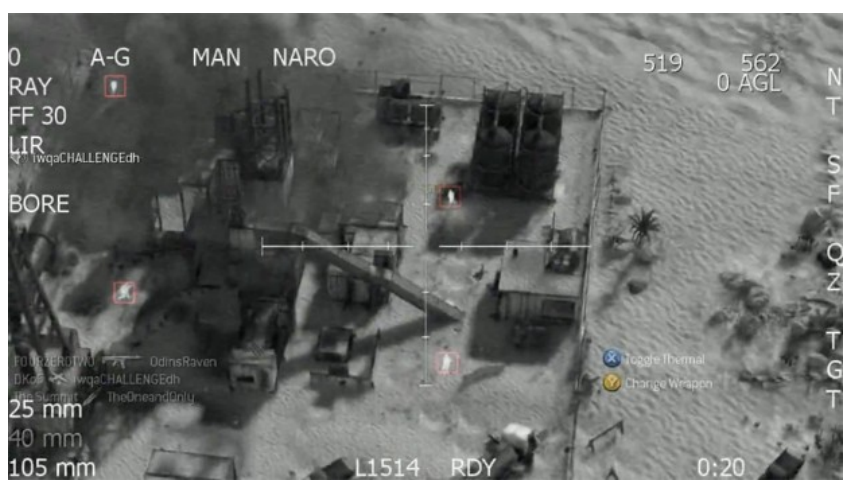

Figure 4: A player calling in an airstrike in Call of Duty 4: Modern Warfare

A critical moment in every round of Super Monday Night Combat is the battle over the annihilator, a Power Weapon that instantly destroys all enemy NPCs and deals a large amount of damage to all enemy players. The annihilator can only be used every five minutes, and in order to activate it a player must first have enough in-game money to pay for it. Then, they have to get to the activation point for the annihilator and remain there for a few seconds, completely vulnerable, in order to set it off.

\subsection{Melee Weapon}

Description - Melee Weapons are hand-to-hand weapons, such as knives or bare hands. These weapons have slow firing rates, but often deal high damage. Melee Weapons discourage firefights that are at close range and reward players for using stealth.

\section{Affordances -}

- Relative strength of the weapon

- Limits on use

Consequences - Melee Weapons allow players to react to Close Blast weapon attacks regardless of their other weaponry. In addition, focusing on using a Melee Weapon frees the player from the constraints of ammunition limits, or allows the player to keep fighting after running out of ammunition.

Level Patterns - Melee Weapons favor small Choke Points and crowded Arenas. They can also be used to quietly make one's way into a Sniper Locations via access, or to sneak one's way through a Flanking Route without giving away the player's location.

$N P C s$ - Rush enemies are usually equipped with Melee Weapons in order make them more threatening at close range. Grunts and Elites sometimes have Melee Weapons in order to discourage players from getting too close to them.

A player with a Melee Weapon can more easily defend themselves against Swarms and Berserkers, as well as sneak up on Snipers and Elites. However, without easy access, the player is vulnerable to Snipers who have the distance advantage, and the player is left with no effective way to engage a Sacrificial or Suicidal enemy.

Examples - In Call of Duty 4: Modern Warfare 2, the player has a combat knife weapon that can always be used to instantly eliminate an enemy. This encourages stealth and ensures that extremely close-range combat will never last long.

Among the many varied Melee Weapons in Team Fortress 2 is the Übersaw, which can be equipped by the medic class. This 
melee weapon has a slower attack time, but charges up the player's special abilities when it hits.

Ratchet and Clank: Up Your Arsenal gives the player two different variations on Melee Weapons. The first, the omniwrench, allows the player to hit enemies that are directly in front of them. The weapon can also be thrown like a boomerang to be used as a Thrown Projectile. The second weapon is the plasma whip, which does more damage, hits enemies in all directions around the player, and has a longer range. However, the use of the plasma whip is limited by ammunition, while the omniwrench has no such limitations.

\subsection{Placed Weapon}

Description - A weapon placed in a stationary location, either by the user or by the level designer. The weapon acts independent of the user according to its own logic, even if the user is no longer present. Placed Weapons are categorized by having a large to medium area of effect, high damage output or a special effect over the area, and some method of activation.

\section{Affordances -}

- The method of activation of the weapon.

- If the weapon has a method through which opponents can deactivate the weapon.

- Any limitations on where the weapon can or cannot be placed.

Consequences - The player gains a sense of control over a larger area.

Level Patterns - Placed Weapons allow a player to easily defend a Choke Point or a Sniping Location through giving an effective way to defend a small area. They also allow a player to cover multiple entrances to a Stronghold simultaneously.

Placed Weapons are ineffective when Flanking Routes or large Arenas allow enemies to simply circumvent the area of effect. They are also not useful if the player needs to assault a Choke Point or Stronghold.

NPCs - It is rare for NPCs to have Placed Weapons, but occasionally they can be found in the hands of Elites and Tanks.

Place Weapons are most effective against Aggressive enemies, whom the player can easily lead into the area of effect of a Placed Weapon. Shielded enemies, however, can often protect themselves against the efforts of a Placed Weapon.

Examples - In Team Fortress 2, the Engineer class can build a sentry gun that will automatically detect and fire upon enemies within its range. Enemies can destroy the turret by attacking it with normal weapons, but the Engineer can keep it alive by continually repairing it. Some areas of levels, such as spawn areas and capture points, cannot have sentry guns built upon them, as specified by the level designer, in order to prevent the weapon from being overpowered.

Throughout the world of Half-Life 2, explosive barrels are scattered about. The player has the ability to pick these barrels up and place them as they please, setting up traps in Choke Points or fortifying their defenses when tasked with guarding a Stronghold.
The Karl character in Super Monday Night Combat has the ability to place a small, hovering robot called "Junior." When an enemy comes within the short range of this weapon, it will follow that enemy until it either makes contact with an enemy or level geometry. Once that happens, it detonates, dealing a high amount of damage to a single target.

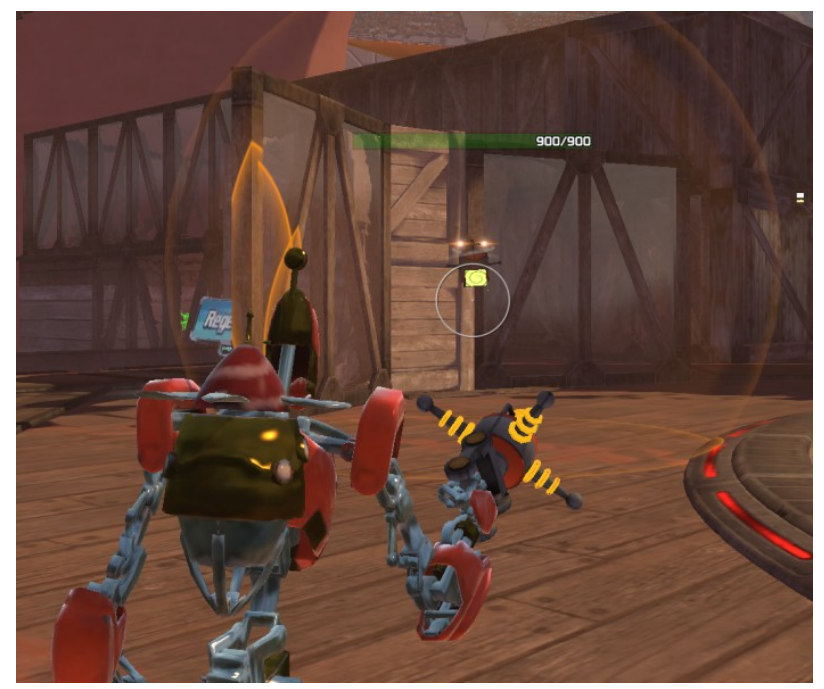

Figure 5: Karl placing a Junior robot in Super Monday Night Combat

\section{EFFECTS ON LEVEL DESIGN}

By forcing the player to use particular weapons in certain parts of a level, the level designer utilizes the relationships between the weapon and level to best control the experience and gameplay.

For example, in the Ravenholm section of Half-Life 2, the player begins the level with a weak Melee Weapon, Sidearm, and Assault Weapon. The player progresses through Arenas and Chokepoints with a numerous number of Grunt and Swarm enemies, resulting in high tension and challenge. Later, the player fights Berserker and Carrier enemies, but acquires a Close Blast weapon and moves into Choke Points where the player has the advantage. The tension and challenge drop to give the player a respite and allow them to learn how to utilize the weapon. As the player proceeds, the level patterns become more Arenas and Split Levels, forcing the player to use weapons accordingly, bringing the challenge and tension back up for the climax of the level.

In multiplayer levels, weapon placement allows the level designer to direct players. The designer can hint at what weapons are best suited for a certain area, force players to carry an unsuitable weapon across an area to get somewhere where that weapon is more useful, or even make it more difficult to use a particular weapon from a particular location.

The multiplayer level Blood Gulch in Halo has Sniping Weapons atop each base at the ends of the map, overlooking large amount of the level and subtly hinting at the advantageous Sniper Position. A Power Weapon, the rocket launcher, is placed in the center of the map, forcing players to travel a long distance and expose themselves in order to procure the weapon. 


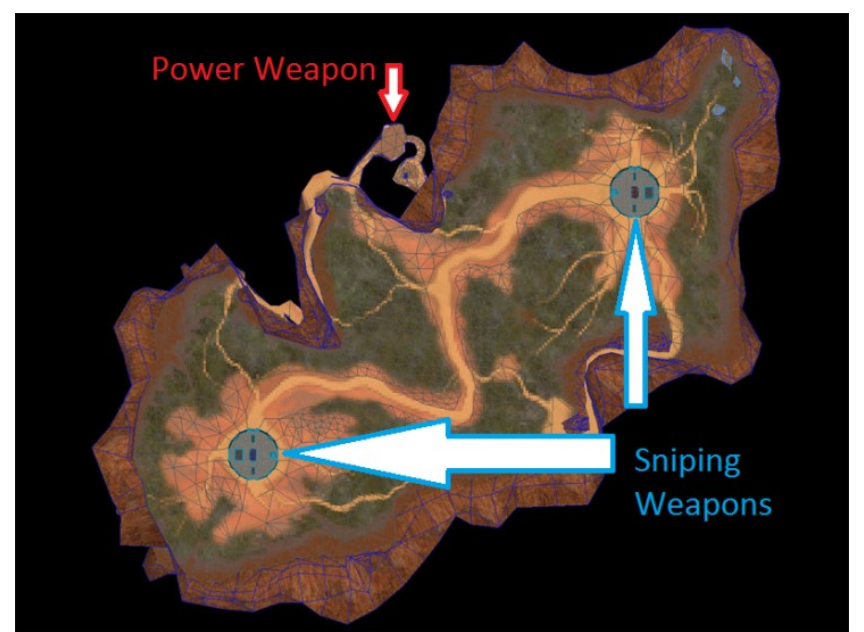

Figure 6: The multiplayer level Blood Gulch in Halo

\section{FURTHER WORK}

Though we have developed a framework of patterns for weapon design, our work is merely an observational study. For further study, we would like to delve deeper into these patterns. By refining the patterns that we have already identified, we could gain a greater understanding of the underlying effects of different design decisions and how to best utilize them to achieve design goals.

\section{GAMES CITED}

Call of Duty 4: Modern Warfare 2, Infinity Ward, 2009

Goldeneye 007, Rare Ltd., 1997
Half-Life 2, Valve Software, 2004.

Halo, Bungie Software, 2001.

Halo 3, Bungie Software, 2007.

Ratchet and Clank: Up Your Arsenal, Insomniac Games, 2004.

Super Monday Night Combat, Uber Entertainment, 2012

Team Fortress 2, Valve Software, 2007

\section{REFERENCES}

[1] Björk, S. and Holopainen, J. "Patterns in Game Design." Charles River Media. 2004.

[2] Björk, S. "Gameplay Design Pattern Collection." August 8, 2011. http://gdp2.tii.se

[3] Hullett, K. and Whitehead, J. "Design Patterns in FPS Levels." In Proceedings of the 5th International Conference on Foundations of Digital Games (FDG 2010). Monterey, California, USA. June 19-21, 2010.

[4] Larsen, S. "Character Advancement in the First-Person Shooter Genre." MIT Thesis, SMU Guildhall, Dallas, TX. 2009.

[5] Rivera, G., Hullett, K. and Whitehead, J. "NPC Design Patterns within the Shooter Genre." To appear in Proceedings of the Workshop on Design Patterns in Games (DPG 2012). Raleigh, North Carolina, USA. May 29, 2012

[6] Weir, G. "Analysis: Character Progression in F.E.A.R." Gamasutra. April 11, 2011. http://www.gamasutra.com/view/news/33878/Analysis_Character _Progression_in_FEAR.php 